\title{
The Margulis Invariant of Isometric Actions on Minkowski (2+1)-Space
}

\author{
William M. Goldman \\ University of Maryland, College Park MD 20742 USA
}

\begin{abstract}
Let $\mathbb{E}$ denote an affine space modelled on Minkowski $(2+1)$-space $\mathbb{E}$ and let $\Gamma$ be a group of isometries whose linear part $\mathbb{L}(\Gamma)$ is a purely hyperbolic subgroup of $\mathrm{SO}^{0}(2,1)$. Margulis has defined an invariant $\alpha: \Gamma \rightarrow \mathbb{R}$ closely related to dynamical properties of the action of $\Gamma$. This paper surveys various properties of this invariant. It is interpreted in terms of deformations of hyperbolic structures on surfaces. Proper affine actions determine deformations of hyperbolic surfaces in which all the closed geodesics lengthen (or shorten). Formulas are derived showing that $\alpha$ grows linearly on along a coset of a hyperbolic one-parameter subgroup. An example of a deformation of hyperbolic surfaces is given along with the corresponding Margulis space-time.
\end{abstract}

\section{Introduction}

In 1983, Margulis found the first examples of properly discontinuous actions of nonamenable groups by affine transformations. He used an invariant of affine actions similar to the marked length spectrum of a Riemannian manifold. However, this invariant comes with a well-defined sign which reflects the dynamics of the action. In this paper we discuss this invariant and its relationship with the deformation theory of hyperbolic Riemann surfaces.

In his 1990 doctoral thesis [8], Drumm found an explicit geometric construction of such groups using polyhedra called crooked planes. He showed that the classical construction of Schottky groups can be implemented for isometries of Minkowski space. We give a simple example of Drumm-Schottky groups corresponding to the deformations of hyperbolic structures $\Sigma_{l}$ on a triply-punctured sphere where all three boundary components have equal length $l>0$.

By combining Drumm's construction with properties of the Margulis invariant, we deduce as a corollary that as $l$ increases, every closed geodesic on $\Sigma_{l}$ lengthens.

We begin with a general discussion of affine spaces and their automorphisms. Next we specialize to orientation-preserving future-preserving isometric actions on (2+1)-dimensional Minkowski space, to define the Margulis invariant for affine deformations of purely hyperbolic subgroups of $\mathrm{SO}^{0}(2,1)$. Then we interpret this invariant in terms of deformation theory of Fuchsian groups, with a simple example of a deformation of hyperbolic structures on a triply-punctured sphere with equal boundary lengths. 
I thank Herbert Abels, Virginie Charette, Todd Drumm, François Labourie, Grisha Margulis, and Scott Wolpert for helpful conversations. This work has been supported by grant DMS-9803518 from the National Science Foundation.

\section{Affine representations}

Recall that an affine space is a space $\mathbb{E}$ equipped with a simply transitive action of a vector group, that is, the additive group of a real vector space $V$. For $v \in V$, let

$$
\begin{aligned}
\tau_{v}: \mathbb{E} & \longrightarrow \mathbb{E} \\
x & \longmapsto x+v
\end{aligned}
$$

denote translation by $v$. If $x \in \mathbb{E}$ and $v \in V$, the image of translating $x$ by $v$ will be denoted $x+v$. As such an affine space inherits the structure of a smooth manifold with a flat torsionfree affine connection. This structure is invariant under the action of $V$, and the action of $V$ canonically identifies each tangent space $T_{x} \mathbb{E}$ with the vector space $V$. Furthermore the connection is geodesically complete, and an affine space can be alternatively defined as a 1-connected smooth manifold with a geodesically complete flat torsionfree affine connection.

Let $\mathbb{E}, \mathbb{E}^{\prime}$ be affine spaces with underlying vector spaces $V, V^{\prime}$ and actions $\tau, \tau^{\prime}$ respectively. A mapping $h: \mathbb{E} \longrightarrow \mathbb{E}^{\prime}$ is affine if and only if for each $v \in V$, there exists $v^{\prime} \in V^{\prime}$ such that the diagram

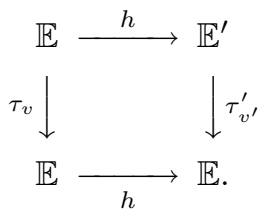

Clearly $v^{\prime}$ is uniquely determined by $v$, and the correspondence

$$
\begin{aligned}
& V \longrightarrow V^{\prime} \\
& v \longmapsto v^{\prime}
\end{aligned}
$$

is a linear map

$$
\mathbb{L}(h): V \longrightarrow V^{\prime}
$$

called the linear part of $h$. Under the identification of $V$ with the tangent spaces $T_{x}(\mathbb{E})$, the linear part $\mathbb{L}(h)$ identifies with the differential $(d h)$ : $T_{x} \mathbb{E} \longrightarrow T_{h(x)} \mathbb{E}$. The group of affine automorphisms $\mathbb{E} \longrightarrow \mathbb{E}$ will be denoted Aff $(\mathbb{E})$ and the linear part defines a homomorphism

$$
\mathbb{L}: \operatorname{Aff}(\mathbb{E}) \longrightarrow \mathrm{GL}(V)
$$


with kernel the translation group $V$. In particular $\operatorname{Aff}(\mathbb{E})$ is the semidirect product $\mathrm{GL}(V) \ltimes V$

Let $\Gamma$ be a group and $\phi: \Gamma \longrightarrow \operatorname{Aff}(\mathbb{E})$ a homomorphism. Then the composition $\Phi=\mathbb{L} \circ \phi: \Gamma \longrightarrow \operatorname{GL}(V)$ is a linear representation, defining a $\Gamma$-module structure on $V$, which we denote $V_{\Phi}$. We call $\Phi$ the linear part of $\phi$. The translational part $u: \Gamma \longrightarrow V$ of $\phi$ is defined by

$$
\phi(\gamma)(x)=\Phi(\gamma)(x)+u(\gamma)
$$

and is a 1-cocycle of $\Gamma$ with values in $V_{\Phi}$, that is,

$$
u\left(\gamma_{1} \gamma_{2}\right)=u\left(\gamma_{1}\right)+\Phi\left(\gamma_{1}\right) u\left(\gamma_{2}\right) .
$$

Using the semidirect product decomposition $\operatorname{Aff}(\mathbb{E})=\mathrm{GL}(V) \ltimes V$, we write $\phi=(\Phi, u)$. We call $\phi$ an affine deformation of $\Phi$.

Conjugation of $\phi$ by translation $\tau_{v}$ gives a new representation $\tau_{v} \circ \phi \tau_{v}$ with linear part $\Phi$ as before, but with translational part $u+\delta_{\Phi} v$ where $\delta_{\Phi} v$ : $\Gamma \longrightarrow V$ is the coboundary

$$
\delta_{\Phi} v: \gamma \longmapsto v-\Phi(v) .
$$

In [18] this cohomology class is called the radiance obstruction and its properties are explored in $[19,20]$. In particular for a given linear representation $\Phi: \Gamma \longrightarrow \mathrm{GL}(V)$, the collection of translational conjugacy classes of its affine deformations identifies with the cohomology $H^{1}\left(\Gamma, V_{\Phi}\right)$.

$c_{\phi}=0$ if and only if $\phi$ fixes a point. For $c_{\phi}=0$ if and only if $\phi$ is conjugate by a translation $\tau_{v}$ to an affine deformation with zero translational part (a linear representation), and thus $\phi$ fixes $v$.

A complete affine manifold is a quotient of $\mathbb{E}$ by a discrete group of affine transformations. The basic problem is to find criteria for the properness of an affine action; unlike in Riemannian geometry a discrete subgroup of Aff( $(\mathbb{E})$ need not act properly on $\mathbb{E}$. This question is discussed in the influential survey article of Milnor [26]. For amenable groups, the question essentially reduces to difficult questions in representation theory of Lie algebras (see [17] for the three-dimensional case). In particular, Milnor raised the question whether a nonamenable group can act properly on $\mathbb{E}$, and Margulis [23,24] found the first examples in dimension 3. (Products with 3-dimensional examples provide examples in all dimensions $\geq 3$.) For further general information see the survey articles by Abels [1] as well as [6].

Three-dimensional compact complete affine manifolds were classified in Fried-Goldman [17], and shown to be finite quotients of solvmanifolds, nilmanifolds and tori. For this reason we assume that $\Gamma$ is nonsolvable. In that case the linear holonomy of a proper affine action of $\Gamma$ preserves an indefinite quadratic form on $V([17])$ and we assume (by possibly passing to a finiteindex subgroup) that $\Phi(\Gamma)$ lies in the identity component $G=\operatorname{SO}^{0}(2,1)$ of the orthogonal group of Minkowski $(2+1)$-space. The corresponding quotient 
$\mathbb{E} / \phi(\Gamma)$ has a flat Lorentz metric. Furthermore the holonomy homomorphism $\Phi: \Gamma \longrightarrow \mathrm{SO}^{0}(2,1)$ is an isomorphism onto a discrete subgroup [17].

We henceforth assume that $\Phi: \Gamma \longrightarrow \mathrm{GL}(V)$ is a Fuchsian representation, that is an isomorphism onto a discrete subgroup of $\mathrm{SO}^{0}(2,1)$

\section{Lorentzian geometry}

Minkowski $(2+1)$-space is an affine space where the translation group $V$ is given the structure of a nondegenerate symmetric bilinear form of index 1 . To indicate this additional structure, we denote it by $\mathbb{E}^{2,1}$. Specifically we consider $V=\mathbb{R}^{3}$ with the bilinear form

$$
\mathbb{B}(v, w)=v_{1} w_{1}+v_{2} w_{2}-v_{3} w_{3} .
$$

The bilinear form $\mathbb{B}$ defines a Lorentz metric on $\mathbb{E}$, which is invariant under the translations of $\mathbb{E}$ (or equivalently parallel with respect to the flat torsionfree connection defined by the affine structure of $\mathbb{E}$ ). Minkowski space $\mathbb{E}^{2,1}$ can be characterized (up to isometry) as a simply connected geodesically complete flat Lorentz 3 -manifold. The isometry group $\operatorname{Iso}\left(\mathbb{E}^{2,1}\right)$ is the subgroup $\mathbb{L}^{-1}(\mathrm{O}(2,1))=\mathrm{O}(2,1) \ltimes V$ with identity component $\operatorname{Iso}^{0}\left(\mathbb{E}^{2,1}\right)=$ $\mathbb{L}^{-1}\left(\mathrm{SO}^{0}(2,1)\right)=\mathrm{SO}^{0}(2,1) \ltimes V$.

Choose a component $\mathfrak{N}_{+}$of the complement of 0 in the nullcone

$$
\mathfrak{N}=\{v \in V \mid \mathbb{B}(v, v)=0\} .
$$

The subset of $\mathrm{O}(2,1)$ preserving orientation and the component $\mathfrak{N}_{+}$is the identity component $G=\mathrm{SO}^{0}(2,1)$ of $\mathrm{O}(2,1)$. This group is isomorphic to $\operatorname{PSL}(2, \mathbb{R})$. An element $g \in \mathrm{SO}^{0}(2,1)$ is hyperbolic if it has three distinct eigenvalues; necessarily all eigenvalues are positive, exactly one equals 1 and the other two eigenvalues are reciprocal. Following Margulis [23], we order them as $\lambda(g)<1<\lambda(g)^{-1}$.

Choose an eigenvector $\mathbf{x}^{-}(g) \in \mathfrak{N}_{+}$for $\lambda(g)$ and an eigenvector $\mathbf{x}^{+}(g) \in$ $\mathfrak{N}_{+}$for $\lambda(g)^{-1}$, respectively. Then there exists a unique eigenvector $\mathbf{x}^{0}(g)$ for $g$ with eigenvalue 1 such that:

- $\mathbb{B}\left(\mathrm{x}^{0}(g), \mathrm{x}^{0}(g)\right)=1$

- $\left(\mathrm{x}^{-}(g), \mathrm{x}^{+}(g), \mathrm{x}^{0}(g)\right)$ is a positively oriented basis.

Notice that $\mathrm{x}^{0}\left(g^{-1}\right)=-\mathrm{x}^{0}(g)$.

Let $\phi=(\Phi, u): \Gamma \longrightarrow$ Aff $(\mathbb{E})$ be an affine deformation of a linear representation $\Phi: \Gamma \longrightarrow \operatorname{SO}^{0}(2,1)$, such that for each $1 \neq \gamma \in \Gamma$, the image $\Phi(\gamma)$ is hyperbolic. The Margulis invariant is the function

$$
\begin{aligned}
\alpha_{\phi}: \Gamma & \longrightarrow \mathbb{R} \\
\gamma & \longmapsto \mathbb{B}\left(\mathrm{x}^{0}(\Phi(\gamma)), u(\gamma)\right) .
\end{aligned}
$$


$\alpha_{\phi}(\gamma)$ depends only on the translational conjugacy class of $\phi$ and the conjugacy class of $\gamma$ in $\Gamma$. Furthermore Todd Drumm and the author have proved [16]:

Theorem 31 Let $\Phi$ be as above. Then two affine deformations $\phi, \phi^{\prime}$ of $\Phi$ are conjugate if and only if $\alpha_{\phi}=\alpha_{\phi^{\prime}}$.

The Margulis invariant relates to restrictions to cyclic subgroups. Consider momentarily the case when $\Gamma$ is cyclic, generated by $\gamma$. Then the coefficient module $V_{\Phi}$ decomposes into the three invariant lines

$$
V_{\Phi}=\mathrm{x}^{0}(\Phi(\gamma)) \mathbb{R} \oplus \mathrm{x}^{+}(\Phi(\gamma)) \mathbb{R} \oplus \mathrm{x}^{-}(\Phi(\gamma)) \mathbb{R}
$$

The projection $V_{\Phi} \longrightarrow \mathrm{x}^{0}(\Phi(\gamma)) \mathbb{R}$ induces a cohomology isomorphism

$$
H^{1}\left(\Gamma, V_{\Phi}\right) \stackrel{\cong}{\longrightarrow} H^{1}\left(\Gamma, \times^{0}(\Phi(\gamma)) \mathbb{R}\right) \cong \mathbb{R} .
$$

Then $\alpha_{\phi}(\gamma)$ is the image of the cohomology class $c_{\phi}=[u] \in H^{1}\left(\Gamma, V_{\Phi}\right)$. (Equivalently it is the inverse image under the cohomology isomorphism induced by inclusion $\mathrm{x}^{0}(\Phi(\gamma)) \hookrightarrow V_{\Phi}$.)

A key observation is that for cyclic groups, the sign of $\alpha$ is independent of the choice of generator since $\alpha\left(h^{n}\right)=|n| \alpha(h)$. Furthermore, when $\Gamma=\mathbb{R}$, so that $\Phi(t)=\exp (t \eta)$ for an element $\eta$ of the Lie algebra of $\operatorname{Iso}^{0}\left(\mathbb{E}^{2,1}\right)$, there exists $\alpha_{1} \in \mathbb{R}$ (the $\mathrm{x}^{0}(g)$-component of the translational part of $\eta$ ) such that

$$
\alpha(\exp (t \eta))=\alpha_{1}|t| .
$$

We call $\alpha_{1}$ the infinitesimal Margulis invariant of the hyperbolic one-parameter subgroup $\exp (t \eta)$. Thus $\alpha$ grows linearly on cyclic subgroups and one-parameter subgroups. We say that $\phi$ is positive (respectively negative) if and only if $\alpha_{\phi}(\gamma)>0$ (respectively $\alpha_{\phi}(\gamma)<0$ ) for every $\gamma \in \Gamma-\{1\}$.

If $\phi$ is a proper affine deformation, then $\mathbb{E} / \phi(\Gamma)$ is a complete flat Lorentz manifold $M$ with fundamental group $\pi_{1}(M) \cong \Gamma$. Suppose $\gamma \in \Gamma$ is represented by a hyperbolic element. Then there exists a unique (necessarily spacelike) closed geodesic in $M$ in the free homotopy class corresponding to $\gamma$, and $\left|\alpha_{\phi}(\gamma)\right|$ is its Lorentzian length.

In general the $\mathbb{R}$-valued class function $\alpha_{\phi}$ on $\Gamma$ expresses the maps

$$
H^{1}\left(\Gamma, V_{\Phi}\right) \longrightarrow H^{1}\left(\langle\gamma\rangle, V_{\Phi}\right) \cong \mathbb{R}
$$

induced on cohomology by restriction to cyclic subgroups $\langle\gamma\rangle \subset \Gamma$.

\section{Deformation theory}

Let $\mathrm{T} G$ denote the tangent bundle of $G$, regarded as a Lie group. Specifically, let $\mathbb{R}[\varepsilon]$ be the ring of dual numbers, that is the truncated polynomial 
$\mathbb{R}$-algebra with one generator $\varepsilon$ subject to the relation $\varepsilon^{2}=0$. Then $\mathrm{T} G$ identifies with the group $\operatorname{PSL}(2, \mathbb{R}[\varepsilon])$ of $\mathbb{R}[\varepsilon]$-points of the algebraic group $\operatorname{PSL}(2)$. Explicitly, an element of $\operatorname{PSL}(2, \mathbb{R}[\varepsilon])$ is given by

$$
X=X_{0}+\varepsilon X_{1}= \pm\left[\begin{array}{ll}
a_{0}+\varepsilon a_{1} & b_{0}+\varepsilon b_{1} \\
c_{0}+\varepsilon c_{1} & d_{0}+\varepsilon d_{1}
\end{array}\right]
$$

with $\operatorname{det}(X)=1+0 \varepsilon=\left(a_{0} d_{0}-b_{0} c_{0}\right)+\left(d_{0} a_{1}-c_{0} b_{1}-b_{0} c_{1}+a_{0} d_{1}\right) \varepsilon$. Thus $X_{0} \in \operatorname{PSL}(2, \mathbb{R})$ and $X_{1}\left(X_{0}\right)^{-1} \in \mathfrak{s l}(2, \mathbb{R})$.

The ring homomorphism $\Phi: \mathbb{R}[\varepsilon] \longrightarrow \mathbb{R}$ with kernel $(\varepsilon)$ induces a group homomorphism T $G \longrightarrow G$ (corresponding to the fibration $\Pi: \mathrm{T} G \longrightarrow G$ ) with kernel $T_{e} G \cong \mathfrak{g} \cong \mathfrak{s l}(2, \mathbb{R})$. The diagram

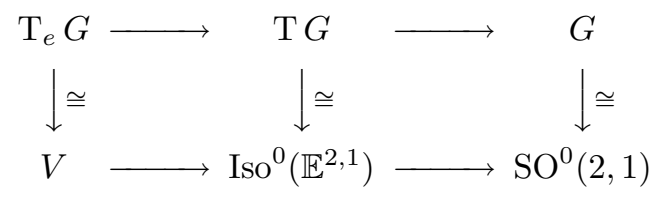

is commutative, with vertical maps isomorphisms. Furthermore the extension is split, so that $\mathrm{T} G$ equals the semidirect product $G \ltimes \mathfrak{g}$, where $G$ acts on $\mathfrak{g} \cong \mathfrak{s l}(2, \mathbb{R})$ by its adjoint representation. (Here we identify $\mathfrak{g}$ with the Lie algebra of right-invariant vector fields on $G$ ).

Let $\Phi: \Gamma \longrightarrow G$ be a homomorphism as above. A deformation of $\Phi$ is a path $\Phi_{t}: \Gamma \longrightarrow G$ of homomorphisms varying analytically in $t$ such that $\Phi_{t_{0}}=\Phi$ for some parameter value $t_{0}$. For each $\gamma \in \Gamma$, the velocity vector of the path $\Phi_{t}(\gamma)$ is a tangent vector

$$
\frac{d}{d t} \Phi_{t}(\gamma) \in T_{\Phi_{t}(\gamma)} G
$$

to $G$ at $\Phi_{t}(\gamma)$. Apply (the differential of) right-multiplication by $\Phi_{t}(\gamma)^{-1}$ to obtain a tangent vector at the identity element $e \in G$ :

$$
\dot{\Phi}_{t}(\gamma):=\frac{d \Phi_{t}(\gamma)}{d t} \Phi_{t}(\gamma)^{-1} \in T_{e} G \cong \mathfrak{g}
$$

$\dot{\Phi}_{t}$ defines a cocycle $\Gamma \longrightarrow \mathfrak{g}_{\text {Ad } \circ \Phi_{t}}$ with values in the $\Gamma$-module $\mathfrak{g}_{\text {Ad } \circ \Phi_{t}}$ defined by the adjoint representation $\operatorname{Ad} \circ \Phi_{t}: \Gamma \longrightarrow \operatorname{Aut}(\mathfrak{g})$ :

$$
\begin{aligned}
\dot{\Phi}_{t}\left(\gamma_{1} \gamma_{2}\right) & =\left(\frac{d\left(\Phi_{t}\left(\gamma_{1}\right) \Phi_{t}\left(\gamma_{2}\right)\right)}{d t}\right)\left(\Phi_{t}\left(\gamma_{1}\right) \Phi_{t}\left(\gamma_{2}\right)\right)^{-1} \\
& =\left(\frac{d \Phi_{t}\left(\gamma_{1}\right)}{d t} \Phi_{t}\left(\gamma_{2}\right)+\Phi_{t}\left(\gamma_{1}\right) \frac{d \Phi_{t}\left(\gamma_{2}\right)}{d t}\right)\left(\Phi_{t}\left(\gamma_{2}\right)^{-1} \Phi_{t}\left(\gamma_{1}\right)^{-1}\right) \\
& =\frac{d \Phi_{t}\left(\gamma_{1}\right)}{d t} \Phi_{t}\left(\gamma_{1}\right)^{-1}+\Phi_{t}\left(\gamma_{1}\right)\left(\frac{d \Phi_{t}\left(\gamma_{2}\right)}{d t} \Phi_{t}\left(\gamma_{2}\right)^{-1}\right) \Phi_{t}\left(\gamma_{1}\right)^{-1} \\
& =\dot{\Phi}_{t}\left(\gamma_{1}\right)+\operatorname{Ad}\left(\Phi_{t}\left(\gamma_{1}\right)\right) \dot{\Phi}_{t}\left(\gamma_{2}\right)
\end{aligned}
$$


Furthermore the cocycle tangent to a path $\Phi_{t}: \gamma \longmapsto \eta_{t} \Phi(\gamma) \eta_{t}^{-1}$ induced by conjugation by a path $\eta_{t}$ in $G$ is the coboundary

$$
\dot{\Phi}_{t}=\delta_{t} v_{t}: \gamma \longmapsto v_{t}-\operatorname{Ad}\left(\Phi_{t}(\gamma)\right)\left(v_{t}\right)
$$

where $v_{t}=\frac{d \eta_{t}}{d t} \eta_{t}^{-1}$.

A lift of a homomorphism $\Phi: \Gamma \longrightarrow G$ is a homomorphism $\tilde{\Phi}: \Gamma \longrightarrow \mathrm{T} G$ such that $\Pi \circ \tilde{\Phi}=\Phi$. A deformation $\Phi_{t}$ determines a lift $\tilde{\Phi}_{t}$ by:

$$
\tilde{\Phi}_{t}: \gamma \longmapsto \Phi_{t}(\gamma)+\varepsilon \dot{\Phi}_{t}(\gamma) .
$$

Thus affine deformations of $\Phi: \Gamma \longrightarrow G$ correspond to lifts $\tilde{\Phi}: \Gamma \longrightarrow \mathrm{T} G$, that is to infinitesimal deformations of $\Phi$.

Following [21], define

$$
\begin{aligned}
\ell: G & \longrightarrow \mathbb{R} \\
\gamma & \longmapsto \inf _{x \in \mathbf{H}_{\mathbb{R}}^{2}} d(x, \gamma(x)) .
\end{aligned}
$$

If $\hat{\gamma}$ denotes a preimage in $\operatorname{SL}(2, \mathbb{R})$, then $\ell(\gamma)$ admits the expression:

$$
\ell(\gamma)= \begin{cases}2 \cosh ^{-1}|\operatorname{trace}(\hat{\gamma}) / 2| & \text { if }|\operatorname{trace}(\hat{\gamma})| \geq 2 \\ 0 & \text { if }|\operatorname{trace}(\hat{\gamma})| \leq 2\end{cases}
$$

A Fuchsian representation $\Phi: \Gamma \longrightarrow G$ is determined up to conjugacy by its marked length spectrum, that is, the function $\ell \circ \Phi: \Gamma \longrightarrow \mathbb{R}$. For any hyperbolic surface $S$, and any homotopy class $\gamma \in \Gamma_{1}(S)$, either $\gamma$ corresponds to cusp (a finite-area end of $S$ ) or is represented by a unique closed geodesic. $\ell(\gamma)$ measures the length of this geodesic, and is zero in the case of a cusp.

Let $\Phi_{t}$ be a deformation whose derivative $\dot{\Phi}_{t}$ at $t=t_{0}$ corresponds to an affine deformation $\phi$. The Margulis invariant of $\phi$ is the derivative

$$
\alpha_{\phi}(\gamma)=\left.\frac{d}{d t}\right|_{t=t_{0}} \ell\left(\Phi_{t}(\Gamma)\right) .
$$

See [21] for details.

\section{Properness}

Let $\phi$ be an affine deformation. $\alpha_{\phi}$ can be used to detect nonproperness and properness of the affine action $\phi$.

Theorem 51 (Margulis) Suppose that $\phi$ is an affine deformation such that $\Phi(\Gamma) \subset G$ is purely hyperbolic. If $\phi$ defines a proper action on $\mathbb{E}$, then $\phi$ is either positive or negative. 
This theorem first appeared in Margulis [23]. Other proofs have been given by Drumm [10,11] and Abels [1]. We conjecture that the sign of $\alpha$ is the only obstruction for properness:

Conjecture 51 Suppose $\phi$ is a positive (respectively negative) affine deformation. Then $\phi$ defines a proper affine action of $\Gamma$ on $\mathbb{E}$.

An element $\gamma \in \Gamma$ fixes a point if and only if $\alpha_{\phi}(\gamma)=0$. An affine deformation $\phi$ is free if the corresponding action on $\mathbb{E}$ is free. Let $\gamma \in \Gamma$. Let $c \in H^{1}\left(\Gamma, V_{\Phi}\right)$ be the cohomology class corresponding to $\phi$. Then $\phi(\gamma)$ fixes a point if and only if $\iota_{\gamma}^{*}\left(c_{\phi}\right)$ is zero in $H^{1}\left(\langle\gamma\rangle, V_{\Phi}\right)$, that is if $\alpha_{\phi}(\gamma)=0$. This condition defines a hyperplane $H_{\gamma}$ in $H^{1}\left(\Gamma, V_{\Phi}\right)$. The free affine deformations correspond to points in the complement

$$
H^{1}\left(\Gamma_{0}, V_{\Phi}\right)-\bigcup_{\gamma \in \Gamma} H_{\gamma}
$$

Thus Conjecture 51 asserts that the proper actions form two components (one positive, one negative) inside the moduli space of free actions.

In particular under a positive deformation corresponding cohomology class is positive, the closed geodesics on the corresponding hyperbolic surface are all lengthening. For a negative cohomology class, the closed geodesics are all shortening. For closed hyperbolic surfaces, no such deformations exist in which all the curves shorten (or lengthen). This idea is used in [21] to prove the following theorem of Mess [25]:

Theorem 52 (Mess) Let $\Gamma$ be a closed surface group. Then no Fuchsian $\Phi: \Gamma \longrightarrow G$ admits a proper affine deformation.

An equivalent statement is that the linear holonomy group of a complete flat Lorentz 3-manifold cannot be a cocompact subgroup of $\mathrm{SO}^{0}(2,1)$.

Last year, Labourie [22] extended Mess's theorem to higher dimensions, using a higher-dimensional version of the Margulis invariant:

Theorem 53 (Labourie) Let $\Phi: \Gamma \longrightarrow G$ be a Fuchsian representation where $\Gamma$ is the fundamental group of a closed surface. Let $\varrho: G \longrightarrow \mathrm{GL}\left(\mathbb{R}^{n}\right)$ be an irreducible representation. Then $\left.\varrho\right|_{\Gamma}$ admits no proper affine deformation.

\section{Linear Growth}

Margulis's original proof $[23,24]$ of the existence of proper affine actions estimates the growth of $\alpha$ on a coset of $\Gamma$. (For another proof of the existence of proper affine actions using this technique, see [13]). Let $\|\gamma\|$ denote the word-length of $\gamma$ with respect to a finite set of generators. Recall that $\gamma \in H$ is $\varepsilon$-hyperbolic if $\mathbb{L}(\gamma)$ is hyperbolic and the two null eigenvectors $x^{ \pm}(\mathbb{L}(\gamma))$ (normalized to lie on the Euclidean unit sphere) are separated by at least $\varepsilon$. 
Theorem 61 (Margulis) Let $\phi$ be an affine deformation of a Fuchsian representation $\Phi: \Gamma \longrightarrow G$. Suppose $\varepsilon>0, C>0, h_{0} \in \operatorname{Iso}^{0}\left(\mathbb{E}^{2,1}\right)$ such that for each $\gamma \in \Gamma$,

- $h_{0} \Phi(\gamma)$ is $\varepsilon$-hyperbolic,

- $\left|\alpha\left(h_{0} \phi(\gamma)\right)\right| \geq C\|\gamma\|$.

Then $\Phi(\Gamma)$ acts properly on $\mathbb{E}$.

Charette's thesis [4] contains a partial converse to this statement. Namely, let $\Gamma$ be a Drumm-Schottky group, that is a proper affine deformation constructed by Drumm using a crooked fundamental polyhedron. Then there exist $\varepsilon>0, C>0, \gamma_{0} \in \operatorname{Iso}^{0}\left(\mathbb{E}^{2,1}\right)$ satisfying the above conditions.

In general, $\alpha(\gamma)$ seems to grow roughly linearly with $\|\gamma\|$. By using the $\operatorname{PSL}(2, \mathbb{R}[\varepsilon])$ model, we have computed $\alpha$ on cosets of a hyperbolic oneparameter subgroup of $\operatorname{Iso}^{0}\left(\mathbb{E}^{2,1}\right)$. Recall that the infinitesimal Margulis invariant

$$
\alpha_{1}=\mathbb{B}\left(u(\eta), \mathrm{x}^{0}(\exp (\eta))\right)
$$

of the one-parameter subgroup $\exp (t \eta)$ satisfies the exact formula

$$
\alpha(\exp (t \eta))=\alpha_{1}|t| .
$$

Theorem 62 Let $h_{0}=\left(g_{0}, u_{0}\right) \in \operatorname{Iso}^{0}\left(\mathbb{E}^{2,1}\right)$. Suppose $\eta$ generate a hyperbolic one-parameter subgroup $\exp (t \eta)$ with infinitesimal Margulis invariant $\alpha_{1}$. Then

$$
\begin{aligned}
& \alpha\left(h_{0} \exp (t \eta)\right) \sim C_{+}+t \alpha_{1} \quad \text { as } t \longrightarrow+\infty \\
& \alpha\left(h_{0} \exp (t \eta)\right) \sim C_{-}-t \alpha_{1} \quad \text { as } t \longrightarrow-\infty
\end{aligned}
$$

where

$$
C_{ \pm}=\mathbb{B}\left(u_{0}, g_{0}^{\mp 1} \mathrm{x}^{ \pm}(\exp (\eta)) \otimes \mathrm{x}^{\mp}(\exp (\eta))\right) .
$$

For this calculation the $\operatorname{PSL}(2, \mathbb{R}[\varepsilon])$ model is useful. Let

$$
h_{0}:=\left[\begin{array}{ll}
a_{0} & b_{0} \\
c_{0} & d_{0}
\end{array}\right]+\varepsilon\left[\begin{array}{cc}
a_{1} & b_{1} \\
c_{1} & -a_{1}
\end{array}\right], \eta:=\left[\begin{array}{cc}
1 & 0 \\
0 & -1
\end{array}\right]+\varepsilon\left[\begin{array}{cc}
\alpha_{1} & 0 \\
0 & -\alpha_{1}
\end{array}\right] .
$$

Then we have the exact formula

$$
\alpha\left(h_{0} \exp (t \eta)\right)=\frac{\left(a_{0} a_{1}+c_{0} b_{1}+a_{0} \alpha_{1} t\right) e^{t}+\left(-d_{0} a_{1}+b_{0} c_{1}-d_{0} \alpha_{1} t\right) e^{-t}}{\sqrt{\left(a_{0} e^{t}-d_{0} e^{-t}\right)^{2}+4 b_{0} c_{0}}}
$$

which has asymptotics

$$
\begin{array}{ll}
\alpha\left(h_{0} \exp (t \eta)\right) \sim\left(a_{1}+b_{1} c_{0} / a_{0}\right)+\alpha_{1} t & \text { as } t \longrightarrow+\infty \\
\alpha\left(h_{0} \exp (t \eta)\right) \sim\left(-a_{1}+c_{1} b_{0} / d_{0}\right)-\alpha_{1} t & \text { as } t \longrightarrow-\infty .
\end{array}
$$

The theorem follows by expressing these quantities in terms of $h_{0}$ and $\eta$. 


\section{Triangle Group Deformations}

Let $v_{1}, v_{2}, v_{3}$ be unit-spacelike vectors which are symmetric with respect to an order three automorphism $\sigma \in \mathrm{SO}^{0}(2,1)$ :

$$
v_{1} \stackrel{\sigma}{\longrightarrow} v_{2} \stackrel{\sigma}{\longrightarrow} v_{3} \stackrel{\sigma}{\longrightarrow} v_{1},
$$

for example:

$$
\begin{gathered}
\sigma=\left[\begin{array}{ccc}
-1 / 2 & \sqrt{3} / 2 & 0 \\
\sqrt{3} / 2 & -1 / 2 & 0 \\
0 & 0 & 1
\end{array}\right], \\
v_{1}=\left[\begin{array}{c}
0 \\
\sqrt{1+s^{2}} \\
s
\end{array}\right], v_{2}=\left[\begin{array}{c}
\frac{\sqrt{3}}{2} \sqrt{1+s^{2}} \\
-\frac{1}{2} \sqrt{1+s^{2}} \\
s
\end{array}\right], v_{3}=\left[\begin{array}{c}
\frac{\sqrt{3}}{2} \sqrt{1+s^{2}} \\
-\frac{1}{2} \sqrt{1+s^{2}} \\
s
\end{array}\right] .
\end{gathered}
$$

Then

$$
\mathbb{B}\left(v_{i}, v_{j}\right)= \begin{cases}1 & \text { if } i=j \\ -\left(1+3 s^{2}\right) / 2 & \text { if } i \neq j\end{cases}
$$

and we assume that $s>1 / \sqrt{3}$ so that $v_{i}, v_{j}$ correspond to ultraideal geodesics in $\mathbf{H}_{\mathbb{R}}^{2}$ for $i \neq j$. Define

$$
\begin{aligned}
& p_{1}=v_{1} \otimes v_{2} \\
& p_{2}=v_{2} \otimes v_{3} \\
& p_{3}=v_{3} \otimes v_{1}
\end{aligned}
$$

where $\otimes: V \times V \longrightarrow V$ is the Lorentzian cross-product (see [13-15] for example). Then the triple

$$
\left(v_{1}, p_{1}\right),\left(v_{2}, p_{2}\right),\left(v_{3}, p_{3}\right)
$$

satisfies the criterion given in Drumm-Goldman [14,15] (see also Charette [4]) for the crooked planes $\mathcal{C}\left(v_{i}, p_{i}\right)$ to be pairwise mutually disjoint:

$$
\begin{aligned}
\mathbb{B}\left(p_{j}-p_{i}, v_{i} \otimes v_{j}\right) & -\left|\mathbb{B}\left(p_{j}-p_{i}, v_{i}\right)\right|-\left|\mathbb{B}\left(p_{j}-p_{i}, v_{j}\right)\right| \\
& =\frac{3}{2}\left(1+s^{2}\right)\left(\sqrt{3} s^{2}-|s|\right)>0 .
\end{aligned}
$$

Thus $\mathcal{C}\left(v_{i}, p_{i}\right)$ bound a crooked fundamental domain $\Delta \subset \mathbb{E}$ for the group $\Gamma_{s}$ generated by inversions $\iota_{j}$ in the spacelike lines $l_{j}=p_{j}+\mathbb{R} v_{j}$ (Drumm [8,9,12], see also Charette-Goldman [7]). Hence $\Gamma_{s}$ acts properly on $\mathbb{E}$ for each $s>$ $1 / \sqrt{3}$. (In fact, the disjointness criterion of [15] for asymptotic crooked planes, implies that $\Gamma_{s}$ acts properly for $s=1 / \sqrt{3}$ as well.) Thus the corresponding 
affine deformation acts properly. By Theorem 51 , this affine deformation will be positive or negative for all $s>1 / \sqrt{3}$.

Figure 1 depicts the intersection with a given spacelike plane of the crooked polyhedra bounding a fundamental domain for the original group $\Phi(\Gamma)$ of linear transformations. This group acts properly on the interior of the nullcone, but nowhere else. Figure 2 depicts the crooked tiling arising from the proper affine deformation $\phi$, in which the crooked polyhedra tile all of $\mathbb{E}$. Figure 3 depicts the crooked tiling arising from the parameter value $s=1$; this group is contained in the $(2,4,6)$ Schwarz triangle group and is commensurable with the group generated by reflections in the sides of a regular right hexagon.

This family of proper affine actions corresponds to a deformation $\Phi_{t}$ of Fuchsian groups as follows. Let $\hat{\Gamma}$ denote the free product $\mathbb{Z} / 2 \star \mathbb{Z} / 2 \star \mathbb{Z} / 2$ freely generated by involutions $\iota_{1}, \iota_{2}, \iota_{3}$ and $\Gamma$ its index-two subgroup generated by $\tau_{1}=\iota_{2} \iota_{3}, \tau_{2}=\iota_{3} \iota_{1}, \tau_{3}=\iota_{1} \iota_{2}$ subject to the relation $\tau_{1} \tau_{2} \tau_{3}=I$. We may concretely represent $\hat{\Gamma}$ in $\operatorname{PGL}(2, \mathbb{R})$ by matrices

$$
\Phi_{1}\left(\iota_{1}\right)=\left[\begin{array}{cc}
1 & 0 \\
-2 & -1
\end{array}\right], \Phi_{1}\left(\iota_{2}\right)=\left[\begin{array}{cc}
1 & 2 \\
0 & -1
\end{array}\right], \Phi_{1}\left(\iota_{3}\right)=\left[\begin{array}{cc}
-1 & 0 \\
0 & 1
\end{array}\right]
$$

representing reflections in the geodesics in $\mathbf{H}_{\mathbb{R}}^{2}$ with endpoints (in the upperhalf plane model) $(0,-1),(-1, \infty),(\infty, 0)$ respectively. The quotient $\Sigma_{1}:=$ $\mathbf{H}_{\mathbb{R}}^{2} / \Gamma$ is a triply-punctured sphere with a complete hyperbolic structure of finite area. We define a deformation $\Sigma_{t}$ of complete hyperbolic structures (no longer of finite area) in which every closed geodesic lengthens.

For notational simplicity, we make an elementary change of parameter from $s$ to $t$ by:

$$
t+t^{-1}=1+3 s^{2} \geq 2 .
$$

The parameter interval for $s$ is $[1 / \sqrt{3}, \infty)$ whereas the interval for $t$ is $[1, \infty)$. Define a deformation $\Phi_{t}: \hat{\Gamma} \longrightarrow \operatorname{PGL}(2, \mathbb{R})$ of $\Phi_{1}$ by:

$$
\begin{aligned}
\Phi_{t}\left(\iota_{1}\right) & =\left[\begin{array}{cc}
t & t-1 \\
-t-t^{-1} & -t
\end{array}\right], \\
\Phi_{t}\left(\iota_{2}\right) & =\left[\begin{array}{cc}
t^{-1} & t+t^{-1} \\
1-t^{-1} & -t^{-1}
\end{array}\right], \\
\Phi_{t}\left(\iota_{3}\right) & =\left[\begin{array}{cc}
-1 & -1+t^{-1} \\
1-t & 1
\end{array}\right]
\end{aligned}
$$

for every $t>0$. Each of the generators $\tau_{1}, \tau_{2}, \tau_{3}$ of $\Gamma$ has trace $-\left(t+t^{-1}\right)$. The quotient $\Sigma_{t}=\mathbf{H}_{\mathbb{R}}^{2} / \Gamma_{t}$ is a complete hyperbolic surface whose convex core is a triply punctured sphere (pair-of-pants) whose three geodesic boundary components have length $2 \log t$.

For any nontrivial $\gamma \in \Gamma$ the geodesic length function $\ell\left(\Phi_{t}(\gamma)\right)$ which measures the length of $\gamma$ in $\Sigma_{t}$ is an increasing function of $t>1$. In general 
the length function (or equivalently the traces) depend on the length/trace functions of a finite generating set in a somewhat complicated manner. We summarize this application of Lorentz geometry to hyperbolic geometry:

Theorem 71 Let $S$ be a compact surface-with-boundary whose interior is homeomorphic to a triply-punctured sphere. For each $l>0$, let $S \longrightarrow M_{l}$ be the marked hyperbolic surface with geodesic boundary components each of which has length $l$. For any $\gamma \in \pi_{1}(S)$, let $\ell_{\gamma}$ be the geodesic length function. Then

$$
l \longmapsto \ell_{\gamma}\left(M_{l}\right)
$$

is an increasing function with positive derivative.

\section{References}

1. Abels, H., Properly discontinuous groups of affine transformations, A survey, Geometriae Dedicata (to appear).

2. Abels, H., Margulis, G., and Soifer, G., Properly discontinuous groups of affine transformations with orthogonal linear part, C. R. Acad. Sci. Paris Sr. I Math. 324 (1997), no. 3, 253-258.

3. - On the Zariski closure of the linear part of a properly discontinuous group of affine transformations, SFB Bielefeld Preprint 97-083.

4. Charette, V., Proper actions of Discrete Groups on $2+1$ Spacetime, Doctoral dissertation, University of Maryland (2000).

5. _ and Drumm, T., Signed Lorentzian Displacement for Parabolic Transformations (in preparation).

6. __ Goldman W. and Morrill, M., Complete flat affine manifolds, Proc. A. Besse Round Table on Global Pseudo-Riemannian Geometry (to appear).

7. Charette, V. and Goldman, W., Affine Schottky groups and crooked tilings, in "Crystallographic Groups and their Generalizations," Contemp. Math. 262 (2000), 69-98, Amer. Math. Soc.

8. Drumm, T., Fundamental polyhedra for Margulis space-times, Doctoral Dissertation, University of Maryland (1990).

9. _ Fundamental polyhedra for Margulis space-times, Topology 31 (4) (1992), 677-683.

10. , Translations and the holonomy of complete affine flat manifolds, Math. Res. Letters, 1 (1994) 757-764.

11. _ Examples of nonproper affine actions, Mich. Math. J. 39 (1992), 435442

12. L_ Linear holonomy of Margulis space-times, J.Diff.Geo. 38 (1993), 679691.

13. and Goldman, W. Complete flat Lorentz 3-manifolds with free fundamental group, Int. J. Math. bf 1 (1990), 149-161.

14. Crooked planes, Electronic Research Announcements of the A.M.S. 1 (1), (1995), 10-17.

15. _ The Geometry of Crooked Planes, Topology 38 (2) (1999), 323-351. 
16. _ Length-isospectrality of the Margulis invariant of affine actions, (in preparation).

17. Fried, D. and Goldman, W. , Three-dimensional affine crystallographic groups, Adv. Math. 47 (1983), 1-49.

18. _ and Hirsch, M., Affine manifolds with nilpotent holonomy, Comm. Math. Helv. 56 (1981), 487-523.

19. Goldman, W. and Hirsch, M., The radiance obstruction and parallel forms on affine manifolds, Trans. A.M.S. 286 (1984), 639-649.

20. _ Affine manifolds and orbits of algebraic groups, Trans. A. M. S. 295 (1986), 175-198.

21. Goldman, W. and Margulis, G., Flat Lorentz 3-manifolds and cocompact Fuchsian groups, in "Crystallographic Groups and their Generalizations," Contemp. Math. 262 (2000), 135-146, Amer. Math. Soc.

22. Labourie, F., Fuchsian affine actions of surface groups, J. Diff. Geo. (to appear) 23. Margulis, G. A. , Free properly discontinuous groups of affine transformations, Dokl. Akad. Nauk SSSR 272 (1983), 937-940

24. Complete affine locally flat manifolds with a free fundamental group, J. Soviet Math. 134 (1987), 129-134

25. Mess, G., Lorentz spacetimes of constant curvature, (1990), IHES preprint.

26. Milnor, J. W. , On fundamental groups of complete affinely flat manifolds, Adv. Math. 25 (1977), 178-187

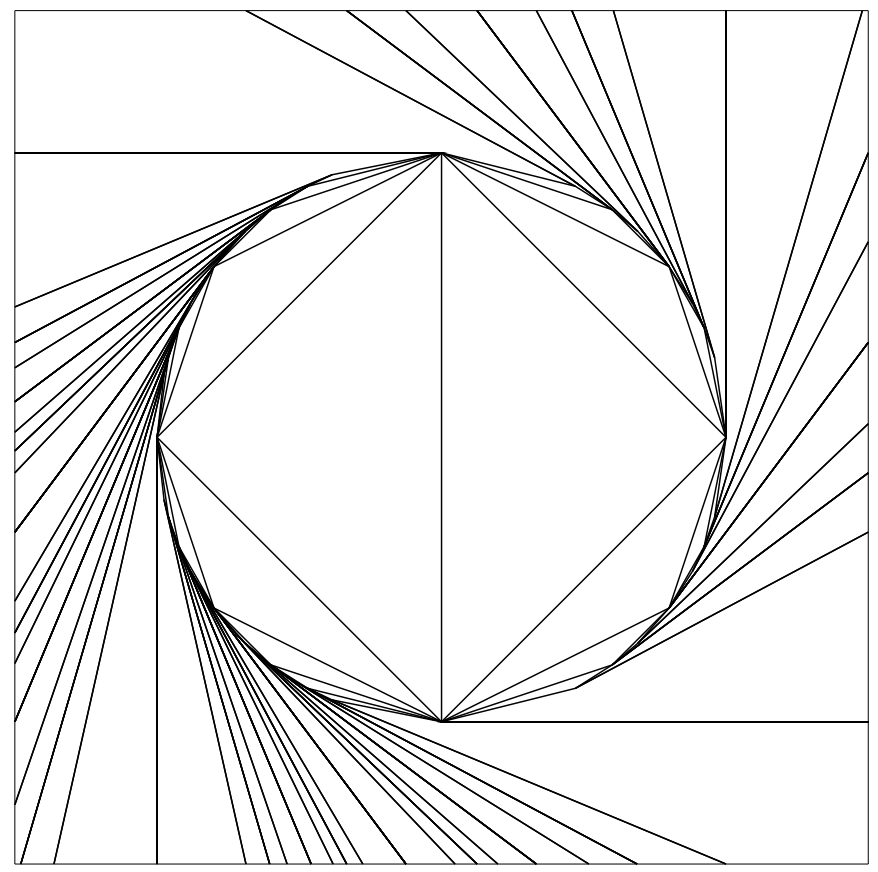

Fig. 1. The linear action of the modular group 


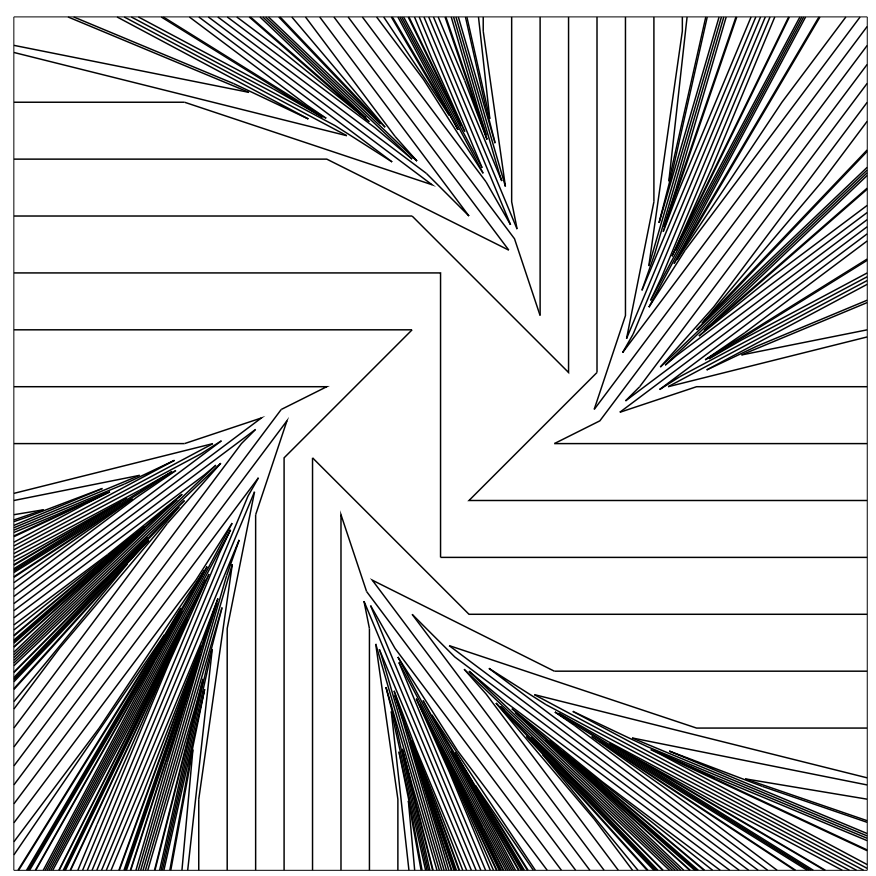

Fig. 2. A proper affine action of the modular group

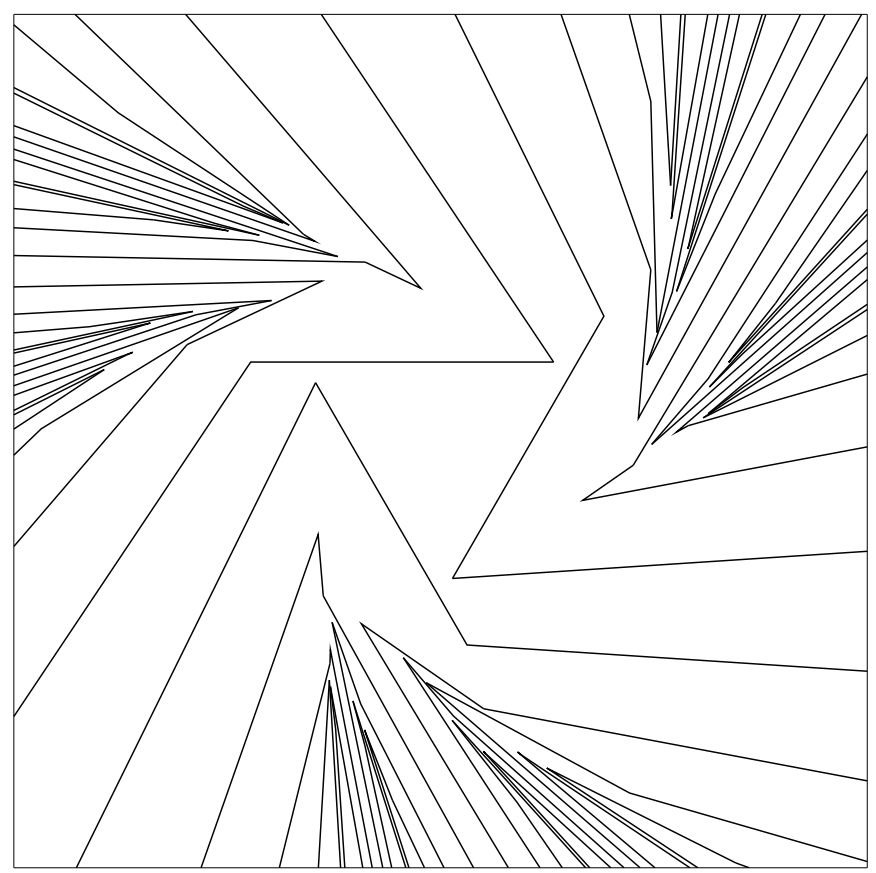

Fig. 3. A proper affine action of an ultraideal triangle group 